\title{
Sulfated Polysaccharide from Marine Red Microalga Porphyridium against SARS-CoV-2 - A Mini-Review
}

\section{Mahadev Gaikwad, Yogesh Pawar, Namrata Gangal, Vinod Nagle and Santanu Dasgupta*}

A20 R and D, RTG, Reliance Industries Ltd., RCP, Ghansoli, Thane, Maharashtra, India

*Corresponding Author: Santanu Dasgupta, A20 R and D, RTG, Reliance Industries Ltd., RCP, Ghansoli, Thane, Maharashtra, India.
Received: January 03, 2022

Published: January 31, 2022

(C) All rights are reserved by Reliance

Industries Ltd.

\begin{abstract}
The recent pandemic of coronavirus, COVID 19, has made researchers to search for novel antidotes against SARS-CoV-2. Utilizing all the available information on this pandemic scientific communities are currently working hard to find antidote. Activity of sulfated polysaccharides is observed on both enveloped and non-enveloped virus against the binding or entry inside the cell. Exopolysaccharides from Porphyridium have antiviral SPs which are active against respiratory coronaviruses. Sanitary items can be coated with these bioactive compounds to prevent COVID-19 infections.
\end{abstract}

Keywords: Porphyridium; COVID-19; Polysaccharides; Carrageenan

\begin{abstract}
Abbreviations
${ }^{\circ} \mathrm{C}$ : Degree Celsius; $\mu \mathrm{g} / \mathrm{ml}$ : Microgram Per Milliliter; COVID-19: Coronavirus; Immunodeficiency 2019; EPS : Exopolysaccharides; GRAS : Generally Regarded as Safe; H1N1: Influenza A Virus Subtype H1N1; hACE-2: Human Angiotensin-Converting Enzyme-2; HBV : Hepatitis B Virus; Hcl : Hydrochloric Acid; hCov OC43 : Human Coronavirus OC43 ; HIV: Human Immunodeficiency Virus; HRV : Human Rhinovirus ; HSV-1 and 2 : Herpes Simplex Virus 1 and 2; MERS-CoV: Middle East Respiratory Syndrome Coronavirus ; MuLV: Murine Leukemia Virus; MuSV: Harvey Murine Sarcoma Virus; RBD: Receptor-Binding Domain; RNA: Ribonucleic Acid; SARS-CoV: Severe Acute Respiratory Syndrome Coronavirus; SPs : Sulfated Polysaccharides ; USFDA: United States Food and Drug Administration; VACV: Vaccinia Virus; VHSV: Viral Hemorrhagic Septicemia Virus
\end{abstract}

\section{Introduction}

In the past few years, more than 30 antiviral drugs are approved for clinical use [1]. Ginsberg., et al. in 1947 reported for the first time that polysaccharides with high molecular weight bear antiviral activity [2]. It took almost 17 years after this report to demonstrate that heparin inhibits herpes simplex virus [3]. Since viruses are getting resistance to the drugs rapidly, there are high demand for developing novel drugs with improved action. Natural sources of such novel drugs can give a promising approach to screen for antiviral compounds. Natural sources like bacteria, algae, fungi plants and animals have potential to produce such anti-viral compounds to fight against these rapidly developing viruses.

Tremendous antiviral potential has been reported from natural compound like polysaccharides especially sulfated polysaccharides (SPs) [4]. Polysaccharides are originally derived from plant sources such as cell walls, seeds, tree exudates and tuber/root $[5,6] \mathrm{s}$; or from animals (chitin, hyaluronan and chondroitin sulfate) $[7,8]$. Additionally, hydrocolloids like gellan, wellan and xanthan are also produced from bacteria and fungi $[9,10]$. Huge number of polysaccharides are produced by different types of algae. These polysaccharides can be of various nature including structural type, storage 
type and some also mucopolysaccharides. Macroalgae also referred to as seaweeds contain tremendous amount of such polysaccharides [11].

Carrageenan; [12], Agaran, Agar [13], Fucoidan [14]; Laminaran and rhamnan sulfate [15] are some of the important antiviral polysaccharides from macroalgae [16]. Glycans, group of polysaccharides, are readily available carbohydrate form in the nature. There is an increasing market demand for naturally extracted polysaccharides to incorporate into food, pharmaceutical products and even cosmetic products. Such a high demand is barely possible to fulfill alone by macroalgae. These macroalgae are cultured in natural resources like marine beaches and their cultivation and harvesting depends purely upon the environmental conditions. To overcome the production uncertainties unicellular microalgae, such as red alga Porphyridium, is a striking alternative. Porphyridium can be grown annually in open and closed systems such as raceway ponds and photobioreactors to achieve high biomass $[17,18]$. This alga was reported for the first time from India by Gaikwad., et al. in 2009 which can be used for bio-refinery approach [19].

Porphyridium is a red microalga whose sulfated polysaccharides encapsulate its' cells. In the growth medium the outer part of this capsule gets solubilized to form exopolysaccharides. These polysaccharides are high molecular weight (5-7 x $\left.10^{6} \mathrm{Da}\right)[20]$ complex sugar molecules composed of $10 \%$ glucuronic acid, $22 \%$ galactose, $24 \%$ glucose and $38 \%$ xylose with minor concentrations of arabinose, rhamnose, and mannose [21]. As these are biocompatible and biodegradable in nature, they are used in variety of applications including medicines, food, and cosmetics [22]. Pujol., et al. in 2007 carried out studies which suggested that there are many sulfated carbohydrates produced by marine algae, blue-green algae and also animals inhibit a number of viruses infecting humans and animals. The main purpose of this article is to recognize the potential of sulfated polysaccharides from marine algae as anti-viral agent in context of currently available data [23]. Our empathetic on COVID-19 pandemic is limited but developing with more research. Information detailed in this work is based on research carried out by other researchers in various viruses.

\section{Coronavirus}

Coronavirus is a virus from the family coronaviridae which can lead to respiratory discomforts like MERS-CoV, SARS-CoV and com- mon cold [24]. Outbreak of COVID-19 occurred in early December 2019 by a novel SARS-CoV-2 virus also known as Betacoronavirus in Wuhan city of China $[25,26]$. Group of coronaviruses are known as enveloped viruses with genetic material in the form of plus stranded RNA. These viruses are the largest in size among RNA viruses. The envelope of this virus display spike proteins in the shape of crown which play a vital role in the attachment with the host cell receptor leading to entry inside the cell $[27,28]$. These viruses can mutate their own genetic material and adapt to the new environment [27].

The role of the Wuhan Seafood Wholesale Market in transmission of the COVID -19 disease is unclear. This market was linked to many initial cases of COVID-19 suggesting that the disease may have spread from animals to humans [29]. A genomic study carried out by Yu., et al. in 2020 provides evidence that the virus was introduced from yet unknown location into the market from where it has spread rapidly. According to them transmission within humans might have happened earlier to this incidence [30]. Spread of the COVID-19 is similar to flu and can be transmitted from infected person to healthy person, principally by physical contacts, while coughing, handshaking, talking with the infected person and sneezing. It was found during SARS-CoV that fomites could carry virus particles up to $96 \mathrm{hrs}$ on its surface and hence can be the same in COVID-19 transmission [31]. Multiple studies have been conducted to find cure and prevention of the disease COVID-19. Sulfated polysaccharides can be one such preventive compound to combat against COVID-19.

\section{Sulfated polysaccharides (SPs)}

Algae are the natural sources of Polysaccharides available with very low cost. These are non-toxic and safe to handle with biodegradable properties and in many cases are biocompatible [32]. Sulfated polysaccharides are macromolecules comprising complex group of polysaccharides exhibiting impeding medical and bioactive properties $[33,34]$. SPs are generally found in saline environment especially in algae, plants and animals. These SPs are the compound which are of increased interest by biomedical and pharmaceutical industries to apply in their products and medicines [35]. According to Aquino., et al. during the evolutionary process, salt adaptation might have developed sulfated polysaccharides in organisms [36]. This was confirmed when Rupia maritime was 
cultivated in both fresh and saline water. Sulfated polysaccharides are chiefly observed only during the saline water cultivation process. They also cultivated Oryza sativa in the condition of salt stress which produced carboxylated polysaccharides instead of SPs. This proposes that the adaptation against salt stress is primarily developed by oppositely charged plant cell wall.

Marine vertebrate and invertebrate animals are principal source of sulfated polysaccharides like heparin [37]. In 2010 Pomin demonstrated that marine invertebrates have capacity to produce sulfated fucans and galactans [38]. Reports indicate absence of SPs in land organisms [39]. Since sulfated polysaccharides are found mainly in marine organisms, they are concentrated more for production of such compounds. Chondroitin sulfate and heparin are two chief sources of active antiviral SPs from marine animals [40].

The SPs blocks the viral attachment to cell surface molecules. The SPs shows similar binding site on CD4 that of HIV-gp120 binding site. Binding of SPs to this lymphocyte inhibit the binding of monoclonal antibodies leading to disruption of CD4-gp120 interaction. SPs shield off the positive charged site on V3 loop of gp120 protein in HIV preventing the viral attachment to cell surface [41].

The SPs anionic nature is involved in blocking of the viral attachment to the host. SPs provide a network of three-dimensional gel over the viral cell surface thus preventing the exposure of cationic domains leading to blockage of the attachment with host cell [42]. Kwon., et al. studied the binding efficiency of SPs to the SARSCoV-2 S-protein in vitro and concluded that these SPs are highly effective in blocking the entry of SARS-CoV-2 to the host cell [43]. Salih., et al. studied marine sulfated polysaccharides using molecular docking and dynamic simulating experiments to find out the interaction of MSPs with RBD of s- protein and hACE- 2 receptors on SARS-CoV-2. They found out that apart from heparin, sulfated galactofucan, sulfated polymannuroguluronate, sulfated Mannan, sulfated heterohamnan and chondroitin sulfate are potential in binding to the receptor sites of SARS-CoV-2 and thus blocking their entry on host cells [44].

Song., et al. 2020 studied four marine sulfated polysaccharides, for their inhibitory activity against SARS-CoV-2. Namely, sea cucumber sulfated polysaccharide (SCSP), fucoidan from brown algae, iota-carrageenan from red algae, and chondroitin sulfate
C from sharks (CS). Among these three SCSP, fucoidan, and carrageenan have shown significant antiviral activities at concentrations ranging from 3.90-500 $\mu \mathrm{g} \mathrm{mL}^{-1}$. Hence, they could be used to treat COVID-19 [45].

Marine green, red and yellow algae are wide sources of SPs. Studies have been carried out on the antibacterial activities of SPs from red alga Gracilaria arcuate on five pathogenic fish bacteria which indicates versatile action of these polysaccharides [46]. Algae based SPs are favored over animal-based SPs due to its vegan source. These SPs are generally excreted out along with mucilaginous exopolysaccharides (EPS). One such alga is Porphyridium belonging to Rhodophyta.

\section{Sulfated polysaccharides from red alga Porphyridium}

Commercial large-scale cultivation of Porphyridium is carried out for its various products [47] (Figure 1). It has many clinically important compounds in the exopolysaccharides. The secretion of exopolysaccharides occurs at the stationary phase during cultivation. Many research studies have demonstrated the potential use of this alga as antiviral, antibacterial and anti-inflammatory agent. High growth rate $\left(0.48 \mathrm{~h}^{-1)}\right.$ [48] and high EPS production (up to 2.5 g/L) [49] makes this alga favorite choice of cultivators (Figure 2.)

Arad, S. and colleagues in 1985 cultivated Porphyridium aeruginum as it is unicellular organism with higher growth rate over other red macroalgae. The phycocolloids also termed as EPS are formed by Porphyridium outside the cells in dissolved conditions which makes the liquid viscous. Since these phycocolloids contain mixture of sulfated sugars they are termed as sulfated polysaccharides [50]. The main composition of polysaccharides from Porphyridium are glucose, xylose, sulfated esters and galactose with minor presence of hexuronic acids [51,52] and fractions of glucuronic acid and galacturonic acid [53]. The polysaccharide produced from Porphyridium is a heteropolymer similar to Carrageenan as sulfated galactans of other red macroalgae. When specific viscosity of polysaccharide was compared with lambda and kappa carrageenans, it was found to be more viscous and hence preferred over others (Figure 3) [51]. 


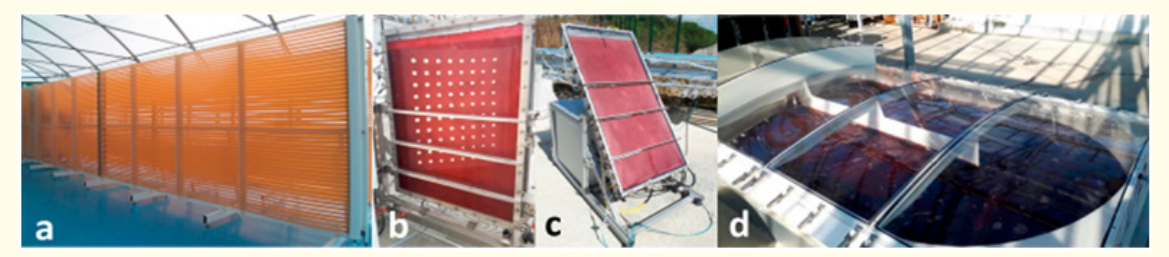

Figure 1: Commercial cultivation of Porphyridium cruentum by various industries: (a) Vertical PBR cultivation in Greensea, Mèze, France; (b) Flat plate vertical photobioreactor with artificial incident light; (c) Inclined flat plate photobioreactor under exposed under sun light (AlgoSolis), (d) Raceway ponds in AlgoSolis with closed transparent cover. Source: Gaignard., et al. 2019.

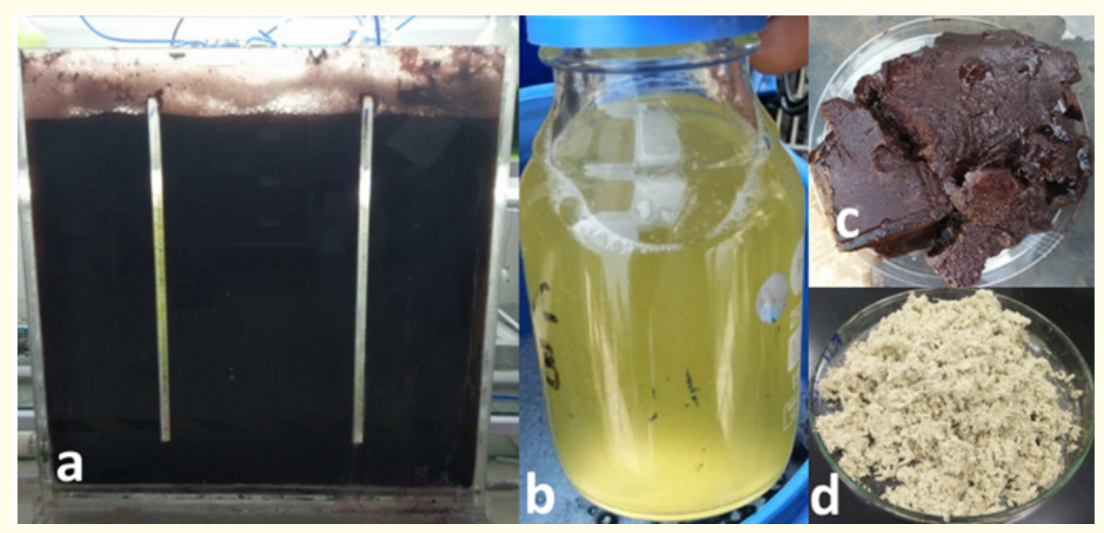

Figure 2: Production of biomass of Porphyridium and EPS in the in-house facility a: cultivation of alga in vertical flat panel photobioreactor, b: Supernatant containing EPS after centrifugation, c: algal biomass as wet slurry, d. exopolysaccharides after drying.

\section{Carrageenans and their importance in clinical trial}

Studies have been carried out by many researchers to validate the clinical benefits of biologically active molecules from red algae to fight against coronaviruses. Carrageenan is one such compound that has antiviral property in human health care. According to Periera L. (2018) carrageenans have ability to inhibit binding of many enveloped and non-enveloped viruses selectively [54].

Certain marine algae were reviewed for bioactivity of their SPs by Jiao, G., et al. in 2011. Though Agarans and carrageenans show structural similarity, agarans have galactans in the 4-linked $\alpha$-galactose residues of the L-series and D-series. High molecular weight carrageenans are classified as per the pattern of sulfation and presence of 3,6-anhydro-galactose on D-units [55].

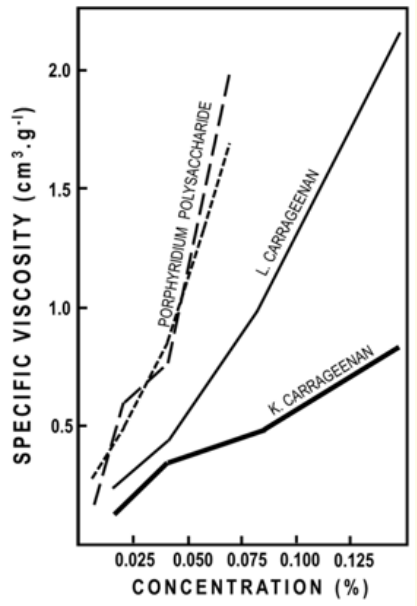

Figure 3: Comparison between viscocities of polysaccharide from Porphyridium and lambda and kappa carrageenans [51]. 
Studies were conducted on patients reporting common cold caused by coronaviruses in 2014. Medical practitioners-initiated treatment with nasal spray containing carrageenan in patients showing early cold symptoms. $50 \%$ of patients were treated with iota-carrageenan nasal spray three times in a day for one week and $50 \%$ patients were supplemented with placebo nasal spray. Patients receiving carrageenan reduced duration of cold than those who received placebo spray. Furthermore, relapses were also accounted in the study which confirm lesser relapse in case of patients treated with carrageenan nasal spray compared to placebo treatment. Viral titer was also found to be lower in patients treated with carrageenan [56]. Morokutti-Kurz, M., (2017) treated patients reporting sore throat with hexylresorcinol, Amylmetacresol/ 2,4-dichlorobenzyl alcohol or carrageenan lozenges as active ingredients. As per the results only carrageenan lozenges were active against H1N1n, HRV8, HRV1a, CoxasackievirusA10 and hCoV OC43 [57]. Similar studies were conducted on patients reporting rhinitis and sinusitis caused by HRv1a and hCoV OC43. Treatment included two active components as xylometazoline $\mathrm{HCl}$ and iotacarrageenan without influencing each other and exhibiting their decongestion and antiviral activities respectively [58].

Carrageenan derived from red algae at the concentration of $400 \mu \mathrm{g} / \mathrm{ml}$ inhibits cell death in case of coronavirus infected patients. Among three types of carrageenans, iota, lambda and kappa, only iota carrageenan demonstrated cell death inhibition at very low concentration of $4 \mu \mathrm{g} / \mathrm{ml}$. other two types require quite high concentration to show positive effects. They also noted that carrageenan pretreated cells remain well protected against coronavirus infection. These results indicate the positive response of carrageenan against coronavirus infection when applied onto solid surface of sanitary material like glove, tissue, masks, cotton swab, etc. [59]. Leibbrandt., et al. carried out a study in 2010 on oseltamivir resistant H1N1 influenza strains which was pandemic in 2009. They used iota carrageenan to find its action against this virus and concluded as a potent inhibitor of Influenza-A virus infection [60].

Recently iota carrageenan from red alga is incorporated into nasal spray Nasitrol ${ }^{\circledR}$ which is launched by Amcyte Pharma in US with the belief that it can resist the transmittance of COVID-19 [61]. According to one clinical trial study, nasal spray containing iota-carrageenan significantly prevented COVID-19 among healthcare workers who were taking care of COVID-19 patients in hospital [62].

\section{Extraction of carrageenan}

A reliable commercial extraction method for kappa, iota and lambda carrageenan was demonstrated by Ramalingam and colleagues in 2003 from marine macroalgae. Extraction of Lambda and iota carrageenans are processed though sun-dried and washed biomass. This is followed by washing dried material in hot environment and filtering the material. The filtrate is evaporated and carrageenans precipitated using alcohol precipitation. The precipitated carrageenans are collected after centrifugation which is dried and powdered [63].

\section{Safety aspects of carrageenan}

In the past 600 years and before, carrageenan has been included in the food applications. Carrageenan is recognized as safe (GRAS) due to its safe use of history since long time by USFDA [64]. Carrageenan is usually found excreted along with exopolysaccharides which contain many other important polysaccharides. The presence of which may also enhance the efficacy of carrageenan.

\section{Exopolysaccharides extraction from Porphyridium}

By using cross flow membrane filtration technique, Balti R., et al. 2018 extracted EPS from Porphyridium. The extraction of crude EPS is also possible from alcohol by concentrating the liquid. Chilled ethanol 90-100\% and centrifuged supernatant are mixed in equal volumes and exposed to $4^{\circ} \mathrm{C}$ overnight. This treatment increases the concentration of EPS. After precipitation, the EPS obtained is dried for further use [65].

Exopolysaccharides from Porphyridium and their antiviral applications

It is evident that animals and algae from marine environment produce sulfated polysaccharides with antiviral activity against many viruses [52]. Exopolysaccharides extracted from various marine red algae are summarized for their antiviral activity by Raposo., et al. in 2013. Porphyridium EPS is known to control viruses like retrovirus, VARICELA ZOSTER VIRUS [24] HSV-1 and 2 [66], MuSV/ MuLV, MuSV-124, VHSV, HBV, AFRICAN SWINE FEVER VIRUS, VACV and VACV-GFP, VESICULAR STOMATITIS VIRUS and ECTROMELIA VIRUS [52]. This makes it a unique application in medicinal field.

Porphyridium EPS is demonstrated to boost immunity in mouse. This is achieved by improving proliferation of spleen cells and de- 
veloping a greater number of killer cells. The antiviral activity of sulphated polysaccharides is determined by the degree of sulfation. In some semisynthetic polysaccharides it is documented that high sulfation of polysaccharides leads to higher antiviral activity [40].

In 2018, Lemos D., et al. extracted and analyzed EPS from Porphyridium and found the coexistence of antiviral carrageenan with agar and xanthum gum. Along with exopolysaccharides Porphyridium also secretes important sugar molecules like $\mathrm{N}$-acetylglucosamine that are known to bind pathogen with slight modification in the saccharide part [67]. The same is also used as a substrate for production of most common sialic acid (N-Acetylneuraminic acid), that act as a surface receptor for most of the influenza viruses [68]. Chemically synthesized clinical molecules containing this sialic are used to produce inhibitors of nuraminidase which is potential against influenza viruses [69].

\section{Conclusion}

From several analytical reports globally carried out on antiviral activity of SPs from Porphyridium, it can be concluded that this molecule can be a great source to combat viral diseases. Its immune boosting property supports the multipurpose use of SPs in COVID-19 pandemic. Marine polysaccharides from seaweeds have great antiviral activities and specifically against SARS-CoV-2. Polysaccharides are believed to interact with the binding of SARS-CoV2 and HSPG or EGFR pathway. Polysaccharides can also changes induce structural changes of SARS-CoV2 RBD protein [70]. As mentioned earlier, coronaviral respiratory disorders are controlled by carrageenans from different biological sources including Porphyridium and hence its role in the current pandemic situation is also expected to be admirable. The spreading rate of COVID-19 is huge and created a serious threat to the world which requires very rapid research for preventing and curing the disease. To add a brick to the building of research we can attempt SPs and carrageenan extracted from Porphyridium in clinical studies and help to reduce viral spread. Due to presence of multiple compounds in the EPS of Porphyridium, this organism and its compounds can change the scenario in treating and preventing COVID-19 pandemic. To find the preventive application, SPs can be coated on respiratory masks and in sanitary products as antiviral agents. This review can help scientists build strong base in developing products for fighting against the virus causing COVID-19 and treating the symptoms.

\section{Conflict of Interest}

There are no conflicts of interest in the current review paper.

\section{Acknowledgements}

Authors acknowledge the support provided by Dr. Ajit Sapre, Head (RandD division), Reliance Industries Ltd for writing this review.

\section{Bibliography}

1. E De Clercq. "Antiviral drugs in current chemical reviews". Journal of Clinical Virology 30 (2004): 115-133.

2. R Ginsberg W., et al. "Inhibition of mumps virus multiplication by a polysaccharide". Proceedings of the Society Experimental Biology and Medicine 66 (1947).

3. AJ Nahmias and S Kibrick. "Inhibitory effect of heparin on herpes simplex virus". Journal of Bacteriology 87.5 (1964): 10601066.

4. G Bedoux., et al. "Antiviral and Cytotoxic Activities of Polysaccharides Extracted from Four Tropical Seaweed Species". Natural Product Communications 12.6 (2017): 807-811.

5. Z Sun., et al. "Aloe Polysaccharides Inhibit Influenza A Virus Infection-A Promising Natural Anti-flu Drug". Frontiers in Microbiology 9 (2018): 2338.

6. H Xirui., et al. "Advances in antiviral polysaccharides derived from edible and medicinal plants and mushrooms". Carbohydrate Polymers 229 (2020): 115548.

7. A Piperno., et al. "Exploring the entrapment of antiviral agents in hyaluronic acid-cyclodextrin conjugates". Journal of Inclusion Phenomena and Macrocyclic Chemistry 93 (2019): 33-40.

8. J Wilson. "The Efficacy of Utilizing Chitosan as an Antiviral Agent in Water Treatmen, Masters dissertation". Emory University (2015).

9. C Gugliandolo., et al. "Antiviral and immunomodulatory effects of a novel bacterial exopolysaccharide of shallow marine vent origin". Journal of Applied Microbiology 116.4 (2014): 10281034. 
10. V Rincão., et al. "Polysaccharides and extracts from Lentinula edodes: structural features and antiviral activity". Virology Journal 15 (2012): 37.

11. M Talyshinsky., et al. "Anti-viral activity of red microalgal polysaccharides against retroviruses". Cancer Cell International 2 (2002): 8 .

12. HHA Gomaa and GA Elshoubaky. "Antiviral Activity of Sulfated Polysaccharides Carrageenan from Some Marine Seaweeds". International Journal of Current Pharmaceutical Review and Research 7.1 (2015): 34-42.

13. A Ahmadi., et al. "Antiviral Potential of Algae Polysaccharides Isolated from Marine Sources: A Review". BioMed Research International (2015).

14. NV Krylova., et al. "The Comparative Analysis of Antiviral Activity of Native and Modified Fucoidans from Brown Algae Fucus evanescens In Vitro and In Vivo". Marine Drugs 18 (2020): 224.

15. C Almeida., et al. "Bioactivities from marine algae of the genus Gracilaria”. International Journal of Molecular Sciences 12.7 (2011): 550-573.

16. C Sasone., et al. "Marine Algal Antioxidants as Potential Vectors for Controlling Viral Diseases”. Antioxidants 9 (2020): 392.

17. GA Lutzu., et al. "Feasibility of attached cultivation for polysaccharides production by Porphyridium cruentum". Bioprocess and Biosystems Engineering 40 (2017): 73-83.

18. S Singh., et al. "Extracellular polysaccharide production in outdoor mass cultures of Porphyridium sp. in flat plate glass reactors". Journal of Applied Phycology 12 (2000): 269-275.

19. M Gaikwad., et al. "On occurrence of the genus Porphyridium nageli: New to India”. Journal of Algal Biomass Utilization 1.1 (2009): 102-106.

20. B Simon., et al. "Degradation of the cell-wall polysaccharide of Porphyridium sp. (Rhodophyta) by means of enzymatic activity of its predator Gymnodium sp. (Pyrrophyta)". Journal of Phycology28 (1992): 460-465.
21. S Garesh., et al. "Characterization of the extracellular polysaccharide of Porphyridium sp.: molecular weight determination and rheological properties". Carbohydrate Polymer 50.2 (2002): 83-189.

22. SB Majee., et al. "Pharmacological, pharmaceutical, cosmetic and diagnostic applications of sulfated polysaccharides from marine algae and bacteria". African Journal of Pharmacy and Pharmacology 11.5 (2017): 68-77.

23. CA Pujol., et al. "Novel dl-galactan hybrids from the red seaweed Gymnogongrus torulosus are potent inhibitors of herpes simplex virus and dengue virus". Antiviral Chemistry and Chemotherapy 13 (2002): 83-89.

24. Burrell J., et al. "Fenner and White's Medical Virology". Academic Press (2016).

25. H Harapan., et al. "Coronavirus disease 2019 (COVID-19): A literature review". Journal of Infection and Public Health 13 (2020): 667-673.

26. R Lu., et al. "Genomic characterization and epidemiology of 2019 novel coronavirus: implications for virus origins and receptor binding". The Lancet 395.10224 (2020): 565-574.

27. A Fip Health. "CORONAVIRUS SARS-CoV-2/ COVID-19 PANDEMIC: Information and interim guidelines for pharmacists and the pharmacy workforce" (2020).

28. SR Weiss and SN Martin. "Coronavirus Pathogenesis and the Emerging Pathogen Severe Acute Respiratory Syndrome Coronavirus". Microbiology and Molecular Biology Reviews 69.4 (2005): 635- 664 .

29. Q Li., et al. "Early transmission dynamics in Wuhan, China, of novel coronavirus-infected pneumonia". The New England Journal of Medicine 32.13 (2020): 1199-1207.

30. W Yu., et al. "Decoding the evolution and transmissions of the novel pneumonia coronavirus (SARS-CoV-2/HCoV-19) using whole genomic data”. Zoological Research 41.3 (2020): 247.

31. U Kramer., et al. "Is there any correlation between severity of epilepsy and cognitive abilities in patients with temporal lobe epilepsy?". European Journal of Neurology 13.2 (2006): 130134. 
32. I Wijesekara., et al. "Biological activities and potential health benefits of sulfated polysaccharides derived from marine algae". Carbohydrate Polymers 84.1 (2011): 14-21.

33. DH Ngo and K Se-Kwon. "Sulfated polysaccharides as bioactive agents from marine algae". International Journal of Biological Macromolecules 62 (2003): 70-75.

34. P Seedevi., et al. "Isolation and characterization of sulfated polysaccharides from Codium tomentosum (J. Stackhouse, 1797) collected from southeast coast of India". Advances in Applied Science Research 4.5 (2013): 72-77.

35. TH Silva., et al. "Marine algae sulfated polysaccharides for tissue engineering and drug delivery approaches". Biomatter 2.4 (2012): 278-289.

36. RS Aquino., et al. "Rising from the Sea: Correlations between Sulfated Polysaccharides and Salinity in Plants". PLOS ONE 6.4 (2011): e18862.

37. HB Nader., et al. "Heparins and heparinoids: occurrence, structure and mechanism of antithrombotic and hemorrhagic activities". Current Pharmaceutical Design 10 (2004): 951-966.

38. VH Pomin. "Structural and functional insights into sulfated galactans: a systematic review". Glycoconjugate Journal 27 (2010): 1-12.

39. J Silva., et al. "Biological activities of the sulfated polysaccharide from the vascular plant Halodule wrightii". Revista Brasileira de Farmacognosia 22.1 (2012): 94-101.

40. T Ghosh., et al. "Focus on antivirally active sulfated polysaccharides: from structure-activity analysis to clinical evaluation". Glycobiology 19.1 (2009): 2-15.

41. C Parish., et al. "A Polyanion Binding Site on the CD4 Molecule. Proximity to the HIV-gp120 Binding Region". Journal of Immunology 145 (1990): 1188-1195.

42. M Andrew and G Jayaraman. "Marine sulfated polysaccharides as potential antiviral drug candidates to treat Corona Virus disease (COVID-19)". Carbohydrate Research 505 (2021): 108326.
43. PS Kwon., et al. "Sulfated polysaccharides effectively inhibit SARS-CoV-2 in vitro". Cell Discovery 6 (2020): 50.

44. A Salih., et al. "Marine Sulfated Polysaccharides as Promising Antiviral Agents: A Comprehensive Report and Modeling Study Focusing on SARS CoV-2". Marine Drugs 19 (2021): 406.

45. S Song., et al. "Inhibitory activities of marine sulfated polysaccharides against SARS-CoV-2". Food and Function 11.9 (2020): 7415-7420.

46. P Adhika., et al. "Bioactivity of Red Seaweed Gracilaria arcuata against Aeromonas hydrophila and Vibrio sp". The Natural Products Journal 8 (2018): 147.

47. C Gaignard., et al. "Screening of marine microalgae: Investigation of new exopolysaccharide producers". Algal Research Biomass, Biofuels and Bioproducts 44 (2019): 101711.

48. Z Csogör., et al. "Growth and product formation of Porphyridium purpureum". Journal of Applied Phycology 13 (2001): 317-324.

49. N Soanen., et al. "Improvement of exopolysaccharide production by Porphyridium marinum". Bioresource Technology 213 (2016): 231-238.

50. V Venugopal. "Sulfated and non-sulfated polysaccharides from seaweeds and their uses: An overview". EC Nutrition 14 (2019): 126-141.

51. S Arad., et al. "The potential of production of sulfated polysaccharides from Porphyridium". Plant and Soil 89 (1985): 117127.

52. DMRMBDMAM Raposo MF. "Bioactivity and applications of sulfated polysaccharides from marine microalgae". Marine Drugs 11 (2013): 233-252.

53. C Gaignard., et al. "New horizons in culture and valorization of red microalgae”. Biotechnology Advances 37 (2019): 193-222.

54. L Periera. "Therapeutic and Nutritional Uses of Algae". A Science publisher's book. CRC Press18 895.

55. G Jiao., et al. "Chemical Structures and Bioactivities of Sulfated Polysaccharides from Marine Algae". Marine Drugs 9 (2011): 196-223. 
56. M Koenighofer., et al. "Carrageenan nasal spray in virus confirmed common cold: individual patient data analysis of two randomized controlled trials". Multidisciplinary Respiratory Medicine 9 (2014): 57.

57. M Morokutti-Kurz., et al. "Amylmetacresol/2,4-dichlorobenzyl alcohol, hexylresorcinol, or carrageenan lozenges as active treatments for sore throat". International Journal of General Medicine 10 (2017): 53-60.

58. C Graf., et al. "Development of a nasal spray containing xylometazoline hydrochloride and iota-carrageenan for the symptomatic relief of nasal congestion caused by rhinitis and sinusitis". International Journal of General Medicine 11 (2018): 275-283.

59. G Andreas and P Eva. "Antiviral composition comprising sulfated polysaccharide". US Patent 0059919 A1 (2011).

60. A Leibbrandt., et al. "Iota-Carrageenan Is a Potent Inhibitor of Influenza A Virus Infection”. PLoS ONE 5.12 (2010): e14320.

61. BioSpace. "biospace.com" (2021).

62. JM Figueroa., et al. "Efficacy of a Nasal Spray Containing IotaCarrageenan in the Postexposure Prophylaxis of COVID-19 in Hospital Personnel Dedicated to Patients Care with COVID-19 Disease". International Journal of General Medicine 14 (2021): 6277-6286.

63. JR Ramalingam., et al. "Commercial scale production of carrageenan from red alga". Seaweed Research and Utilisation 25.1 and 2 (2003): 37-46.

64. P Carthew. "Safety of carrageenan in foods". Environmental Health Perspectives 110.4 (2002): A176-A176.

65. M Ziadi., et al. "Evaluation of the Efficiency of Ethanol Precipitation and Ultrafiltration on the Purification and Characteristics of Exopolysaccharides Produced by Three Lactic Acid Bacteria". BioMed Research International (2018).

66. M Huheihel., et al. "Antiviral effect of red microalgal polysaccharides on Herpes simplex and Varicella zoster viruses". Journal of Applied Phycology 13 (2001): 127-134.
67. Q Xu., et al. "Use of N-Acetyl-D-glucosamine in Preparation of Drugs for Modulating Microorganisms on Mucus Membrane". CA Patent 2539286 A1 (2005).

68. D Lemos., et al. "Rheological characterization of exopolysaccharides from Porphyridium microalgae". in Frontiers in Marine Science Conference Abstract: IMMR'18 International Meeting on Marine Research (2018).

69. JK Chen., et al. "N-Acetylglucosamine: Production and Applications". Marine Drugs 8 (2010): 2493-2516.

70. SIravani and RS Varma. "Important Roles of Oligo- and Polysaccharides against SARS-CoV-2: Recent Advances". Applied Sciences 11 (2021): 3512.

\section{Assets from publication with us}

- Prompt Acknowledgement after receiving the article

- Thorough Double blinded peer review

- Rapid Publication

- Issue of Publication Certificate

- High visibility of your Published work

Website: www.actascientific.com/

Submit Article: www.actascientific.com/submission.php Email us: editor@actascientific.com

Contact us: +919182824667 